\title{
Limitations and Prospects of Skyrmsian Evolutionary Game Theory in the Perspectives of Genotype Phenotype Mapping and Evolutionary Psychology
}

\author{
Chong Ho Yu* \\ Azusa Pacific University, Azusa, California
}

\begin{abstract}
Based upon evolutionary game theory, philosopher of science Brian Skyrms developed certain simulationbased models to explain cultural phenomena for which alternate theories failed to offer plausible explanations. This article aims to examine the preceding claim by unpacking the logical structure of the Skyrmsian theory. Because the author challenges the mathematical model employed by Skyrms, the methodology of this study is analytical rather than mathematical simulation. The Skyrmsian approach is purely phenotypic rather than genotypic. Further, it is an evolutionary generalist and thus omits the psychological mechanisms or the evolutionary histories from which humans emerge. To compensate for the lack of detail in describing psychological mechanisms, mathematical properties such as robustness are introduced in the Skyrmsian approach. This article illustrates how omitting genotypic factors and the interlocking attributes of phenotypic components might pull the Skyrmsian model away from reality. As a model of force that emphasizes the detail of psychological drives (forces), evolutionary psychology can play a vital role in defining input variables for the Skyrmsian approach. At the same time, the latter can function as an evaluation tool to assess the explanatory power of proposed models in terms of robustness. In the era of big data, both evolutionary game theorists and evolutionary psychologists can extract robust behavioral patterns and social dynamics from voluminous data.
\end{abstract}

Keywords: Game theory, Skyrmsian approach, evolutionary psychology

Received: 17 July 2018; Accepted: 18 September 2018; Published: 14 November 2018

\section{INTRODUCTION}

Based upon evolutionary game theory, philosopher of science [1, 2, 3, 4, 5, 6] developed certain simulationbased models to explain cultural phenomena, such as adoption of conventional rules, distributive justice and the formation of social networks, for which alternate theories, such as salient feature theory, rational choice theory, and conventional game theory, could not offer plausible explanations. To be specific, prominent game theorist [7] argued that conventions and norms could arise from recognition of salient features. However, this theory has limitations because in many situations either salient features are totally absent or there is more than one salient feature. Rational choice theory shifts the focus from hints in the environment to human cognitive structure, namely, rationality. However, recently rational choice theory has faced serious challenges because it could lead to irresolvable paradoxes, indeterminacy, and many other problems [8]. For example, in the one-shot prisoner's dilemma, no matter what course of action the other party chooses, apparently the most "rational" choice for a player is defection, which results in receiving a longer jail term than choosing to remain silent under coordination would. Obviously, it does not sound rational at all. According to John Nash, the "beautiful mind" who proposed the Nash equilibrium, any unilateral deviation from an equilibrium

\footnotetext{
${ }^{*}$ Correspondence concerning this article should be addressed to Chong Ho Yu, Azusa Pacific University, Azusa, California. E-mail: cyu@apu.edu

(c) 2018 The Author(s). Published by KKG Publications. This is an Open Access article distributed under a Creative Commons AttributionNonCommercial-NoDerivatives 4.0 International License.
} 
by a player would make that player worse off. However, in the game of divide-the-cake, there are infinitely many solutions that are qualified to be considered an equilibrium: $10 \% / 90 \%, 15 \% / 85 \%, 20 \% / 80 \%, 25 \% / 75 \%$...etc. More importantly, in all these classical theories, it is very difficult to explain how a stable equilibrium could be destabilized by "invasion" and conventions could be changed over several generations because players are said to possess static traits during the course of the game. In brief, in classical game theory players maximize payoff when their identity remains fixed during the course of the game, whereas in evolutionary game theory players are constantly changing their strategies [9]. Other limitations of rational choice theory are also well-documented. Very often our cognitive limitations and imperfect information hinder us from making the right decision [10]. Today many researchers subscribe to the notion proposed by Nobel Laureate [11] that logical assumptions of rationality do not reflect people's actual choices and classical theories failed to take cognitive biases into account. As a remedy, Skyrms proposed a promising approach that seems to work well with low rationality and ambiguous situations.

Further, another appealing feature of the Skyrimsian approach is its spontaneity and naturalness. Inspired by [12] the naturalistic approach, some authors [5] explicitly stated that signals that initially contain no information could spontaneously acquire informational content by evolutionary or learning dynamics. Hence, the central tenet of his approach is: One might use a mindless, self-organizing mathematical model to explain the emergence of social norms and phenomena without invoking any transcendental, hermeneutic, intentional agents, or intelligent designer. Specifically, games played by different participants and social networks could self-assembly themselves through ritualized decisions, modular compositions, or template transfer. In the process of ritualization participants simply follow the custom (ritual) whereas in modular composition simpler games could be merged to form a complicated game. Moreover, in template transfer a game that evolved in one situation could be adapted into another context. The common thread between the preceding methods is that high rationality is not required, and therefore spontaneous self-organization is possible [13].

Could the Skyrimsian approach remediate the shortcomings of rational choice theory and conventional game theory? Is it possible to explain the emergence of norms and social networks by spontaneity and self-organizing mechanisms? Paradoxically, while evolutionary game theorists criticized rational choice theory for its over-reliance on human rationality, justification for evolutionary game theory is still based on logical deduction, which is highly rational in essence. Well-known games in evolutionary game theory include hawk-dove, war of attrition, stag hunt, producer-scrounger, and tragedy of the commons. The solutions of these games are based on logical deduction rather than empirical data. Take the hawk-dove game as an example. The hawk and the dove are referred to as aggressors and peace-makers, respectively. According to evolutionary game theorists, the population could reach the equilibrium point after the hawks and the doves interacted repeatedly. It is stating the obvious, but very few researchers admit that this kind of mathematical model might not applicable to real-life situations [14], even though shortcomings of evolutionary game theory had been revealed by recent research [15]. This article aims to address this research gap by unpacking the Skyrmsian approach in a logical and rational fashion, and also pointing out the limitations of mathematical modeling. Mathematical modeling is an abstraction of reality; however, explaining human behaviors necessitates analysis of concrete situations and example-based modeling. While the author by no means downplay the merits of evolutionary game theory or mathematical modeling, this study offers a balanced view by coping with the advance of big data analytics. In the era of big data, data scientists turn to pattern-seeking by observing numerous concrete examples, in addition to statistical modeling $[16,17]$.

\section{METHODOLOGY}

Following the line of reasoning as stated in the previous section, the author didn't utilize mathematical modeling to examine the claims made by evolutionary game theorists. Rather, the logical structure and philosophical assumptions of the Skyrmsian approach were unpacked; its strength and shortcoming were evaluated with reference to relevant literature [18]. It was argued that evolutionary game theory is descriptive because it illustrates how people would choose. However, the notion that science is descriptive is nothing more than an urban legend. Science is indeed value-laden and our epistemology is often driven by ontological assumptions. Put it bluntly, a naturalistic ontology would lead to an agent-free and naturalistic theory of human behaviors [19]. Apparently, the idea that human norms and social networks emerged from self-organizing mechanisms is implied from a naturalistic framework. The subsequent analysis will let the readers decide whether spontaneity and naturalness of the Skyrmsian model is a bold assumption or a reasonable conclusion, and whether the conclusion yielded from evolutionary game theory is descriptive or prescriptive. 
The starting point adopted by Skyrms is to draw an analogy between R. A. Fishers statistical solution to the sex-ratio problem and the equilibrium of $50 \% / 50 \%$ in the game of divide-the-cake. Like Fisher, Skyrms employed a mathematical model of evolution to explain human behavior. By using Monte Carlo simulations, Skyrms demonstrated how certain behaviors and population structures (e.g., meaningful semantics, the concept of fairness, social networks) could eventually emerge after many generations regardless of what the initial conditions are. This approach does not require the recognition of salient features, nor does it hold a strong assumption of human rationality and common knowledge or a unique Nash equilibrium for any game [18]. More importantly, it gives a plausible account of how a stable system could be invaded under different combinations of topology in the population and under various strategies adopted by players.

There are two distinct characteristics in the Skyrmsian approach. First, it is purely phenotypic rather than genotypic in the sense that it focuses on topology but takes no account of relevant genetic bases for behavioral dispositions as suggested by evolutionary biologists [20]. Second, in contrast to evolutionary psychology, which emphasizes specific psychological factors of human behavior, the Skyrmsian approach is evolutionary generalist; it is a mathematical abstraction instead of an empirical modeling; it entirely omits the psychological mechanisms and evolutionary histories from which humans emerge. The above two features are interrelated in terms of their emphasis on global structure instead of individual attributes as well as on mathematical abstraction instead of empirical detail. To compensate for the lack of detail in explaining human behavior, Skyrms established the merits of his approach by using the criteria of mathematical properties such as representativeness, robustness, flexibility, and parsimony [21].

As it is impossible to exhaust every argument and counter-argument pertaining to the Skyrmsian approach, this paper focuses on the following three aspects. First, the necessity of both genotypic and phenotypic explanations for human behaviors and why the Skyrmsian account may be inadequate will be discussed. Second, by comparing the characteristics of the particularist account offered by evolutionary psychology and the generalist account advocated by evolutionary game theory, the author will discuss how the Skyrmsian approach could remediate the weaknesses of the particularist account of human behavior, though robustness in mathematical modeling could not fully compensate for the lack of detail in describing psychological mechanisms. Last, the particular- ist and the generalist approaches will be compared and contrasted in the context of the theory of force and statistical explanation. It is the conviction of the author that not only are the two approaches not mutually exclusive, but they could also be put together to provide a more thorough presentation of cultural evolution.

\section{GENOTYPES AND PHENOTYPES}

Skyrms built his replicator dynamics on the evolutionary hypothesis in terms of reinforcement by payoff and adaptation. According to Tennant, the Skyrmsian selection process assumes that every individual is (in the relevant phenotypic aspect) exactly like its parent, and that is why the Skyrmsian selection process is considered purely phenotypic [20]. Nonetheless, the modern evolutionary model consists of two components: the theory of natural selection and the theory of Mendelian inheritance. The original evolutionary hypothesis proposed by Charles Darwin stressed environmentally-driven factors, namely, selection pressure on species. The theory of Mendelian inheritance, which emphasizes genetic factors, was proposed by Gregor Mendel in late 19th century and was promoted by de Vries in early $20^{\text {th }}$ century. Although the genetic theory was rejected by the biometric school led by Karl Pearson, by 1918 R. A. Fisher had brought the dispute to a conclusive closure by successfully synthesizing evolutionary theory, biometric methodology, and Mendelianism [22, 23]. The theories of natural selection and Mendelian inheritance explain human behaviors in different domains. Natural selection theory accounts for the variation of a population as a function of the different phenotypes, whereas Mendelianism aims to predict and explain the transmission of genotypes from one generation to the next. In modern evolutionary theory, phenotypes and genotypes are not isolated; rather, genotypes become phenotypes through the process of development. One important aspect of using evolution to explain behaviors is to understand the mechanics of the mapping of genotypes onto phenotypes [24, 25, 26].

In spite of the importance of mapping genotypes onto phenotypes, Skyrms's approach is phenotypic in essence. [13] explicitly pointed out the problem of this narrow focus in the Skyrmsian model:

"The key idea (of replicator dynamics) that Skyrms invokes is that of positive correlation. Skyrms defends the realism of this assumption: If interactions (among players in a replicator dynamic) tend to be among relatives, then interactors will tend to resemble each other. If the phenotype in question is influenced by genes, how far does this observation take us in establishing the plausibility of Skyrms' proposed explanations? An additional question 
that needs to be addressed is whether the different strategies that Skyrms describes are heritable. Do offspring tend to resemble their parents? This might be due to shared genes or to learning and imitation. Since subjects did not act the same in the experiments that Skyrms cites, the question of heritability (at least in the present, if not in the unobservable past) should be tractable."

Further, in Tennant's view [20], real-world populations of social individuals do not always match Skyrms's assumptions. While some important phenotypic traits are heritable, offspring in sexually reproducing species often do not exactly resemble their parents in the phenotypic aspects. Instead, due to genetic recombination, offspring often look different from either one of their parents in traits or behaviors. While Skyrms emphasized how mutants invade a population and subsequently alter the equilibrium, gene recombination in evolution, according to [22], is far more important than mutation in producing heritable variations upon which natural or artificial selection could then act. In other words, the omission of genotypic factors might make the Skyrmsian model unrealistic.

One may argue that, unlike in biological evolution, phenotypic factors should be more important than genotypic components in cultural evolution, such as the formation of conventions and norms. One of the major assumptions in the Skyrmsian model is that biological evolution and cultural evolution are closely analogous. [1] asserted that Darwinian Theory can be imported into the cultural context, in which imitation and learning play an important role. However, Skyrms did not specify how exactly these two types of evolution are related. Is cultural evolution purely phenotypic, as he illustrated? If so, why should we maintain that the theory of biological evolution, which is composed of phenotypic and genotypic elements, is applicable to the theory of cultural evolution, which is highly phenotypic?

Nonetheless, even if only phenotype is taken into consideration, the Skyrmsian selection process may be too simplistic to match the reality. In the past, the phenotype has been held to be highly responsive to the ever-changing environment. Change is inevitable and it occurs incrementally, character by character. However, [27] argued that a phenotypic change in one biological character will almost certainly have an impact on others. Thus, a phenotypic model should not be viewed as a character-environment relationship, but as a system of character integration. Because of the interlocking attribute of biological characters, some phenotypes are intrinsically resistant to evolutionary change. While the conventional approach of phenotype focuses on characters and adaptive evolution, the new structural approach pays attention to adaptive forces in evolution, such as constraints. Although Schwenk's discussion regarding phenotype centers on biological characters, it has important implications for behavioral patterns, social norms, and cultural traits. In the Skyrmsian model, it is assumed that players would change their strategies according to payoff. However, like biological characters, cultural traits are tightly interrelated and thus it is not uncommon that resistance to changes stalls the process of cultural evolution, even at the expense of payoff. As a matter of fact, some conventions and norms in certain older civilizations, though becoming impractical under the wave of globalization, remain intact in spite of low or negative payoff [28]. The Israel-Arab conflict provides another example; this Middle-East crisis is a real-life version of divide-the-cake. In 1947 the United Nations reached a resolution to partition Palestine into a Jewish state and an Arab state. After the 1957, 1963, and 1973 wars and numerous smaller-scaled arms conflicts, adaptive players, theoretically speaking, should have realized that $50 \% / 50 \%$ or $60 \% / 40 \%$ is a more desirable equilibrium than getting nothing at all or paying a high price to keep lands under high uncertainty. In reality, adaptive behaviors according to payoff are found in neither party, which reflects the fact that cultural traits are tightly interlocked and thus resistance against adaptation may be far more common than we thought in the phenotypic cultural evolution.

\section{A. The Particularist Account of Evolutionary Psychol-} ogy and the Generalist Account of Evolutionary Game Theory

Evolutionary psychology is a fairly new discipline, emerging over the past few decades. It is important to point out that although evolutionary psychology is considered a particularist account of human behavior, it is not interested in studying different behaviors in different situations. Rather, its focus is on human nature in the context of how selection pressure shapes a universal cognitive mechanism. The precursor of evolutionary psychology is sociobiology, which also aims to explain human behavior through human nature. Most sociobiologists are genetic determinists, though some are more subtly so than others. For instance, philosopher of science Michael Ruse and the father of sociobiology [29, 30] boldly claimed, "ethics as we understand it is an illusion fobbed off on us by our genes to get us to cooperate". However, most evolutionary psychologists do not focus on genetic explanations at the exclusion of other explanations. In other words, evolutionary psychology is not genetically deterministic. Further, sociobiological accounts often fail in 
generalizing from crucial details of particular ecologies and ontogenies of the species whose behaviors they seek to explain. As a remedy, evolutionary psychology has moved toward a particularist approach to the evolutionary explanation of human behaviors by giving the details of psychological mechanisms [31].

As mentioned before, unlike rational choice theory, which requires a strong assumption of human rationality, evolutionary game theory does not emphasize any universal human cognitive process or human nature. To be explicit, the Skyrmsian mathematical model regards cognitive details as unimportant. Resentment of particularists toward generalists is understandable because it seems that evolutionary game theory falls back to the age of sociobiology. Without some kind of fine-grain story of the biological or psychological mechanism that brings about the behavior in question, the generalist's account could be misused as a form of "naturalistic god of the gaps" [32]. It is understandable that a mathematical approach to the explanation of human behaviors might be considered a kind of "naturalistic God of the gaps" because invoking a highly abstract model could explain too many things. In Christian apologetics, when intelligent design is invoked as the ultimate answer to any phenomenon which has not been explained by science, this approach is mocked as "God of the gaps." By the same token, when the evolutionary dynamic is used while the details of evolution are unexplained, it is said to be the "naturalistic God of the gaps". As a counter-argument, [33] maintained that the particular and the generalist accounts are compatible. What the generalist explanation provides is an explanation for why such behavior was selected in the first place, whereas the particular approach shows how certain specific mechanisms serve to produce certain behaviors.

\section{ANALYSIS OF NATURALNESS, STABILITY, ROBUSTNESS, AND LONG RUN}

Skyrms defended his position by introducing certain mathematical properties, including representativeness, robustness, and flexibility, to explain why psychological state, cultural context, social condition, and other details are irrelevant. Among the three properties, the notion of robustness is the most important one. According to [3], an equilibrium is said to be stable and a model is regarded as robust if the dynamics carry every possible initial state in the interior state space to that equilibrium. If stability and robustness are achieved, a mathematical model can have explanatory value even when we are completely uncertain about the detail of the initial state in the system. Nonetheless, [3] admitted that even a structurally stable model might, after all, be badly mis-specified.

Interestingly enough, a mathematical model that could achieve stable and robust results could be treated as evidence against naturalistic and agent-free explanations. Take Central Limit Theorem as an example. According to Central Limit Theorem, a sampling distribution of any statistics would eventually approximate normality by repeated sampling with replacement, no matter what the initial condition is (it doesn't matter whether the underlying population is normal, skewed, uniform, bimodal, or multi-modal). For some researchers, this amazing "self-organizing" pattern out of seemingly randomness is viewed as a manifestation of divine order instead of spontaneity $[34,35]$.

It is important to point out that, strictly speaking, "stability" and "robustness" are two different concepts. A model demonstrated to be stable is not necessarily also robust. In measurement and statistics, stability is synonymous with temporal reliability and reproducibility [36]. Take the game of divide-the-cake as an example. If the same initial conditions are given, it is expected that all simulations would yield the same or similar results no matter how many simulations are run. In this case no procedure for altering initial conditions is involved. Skyrms is entirely right that a stable model could be totally misspecified. When I use a curved ruler to measure my height a hundred times and all results consistently show that I am six feet tall, no doubt the measurement model is stable, but it does not carry any explanatory value to my physical condition.

In statistics, the term "robustness" can be interpreted literally. Consider this analogy: If a person is robust (strong), he will be immune to hazardous conditions such as extremely cold or extremely hot weather. If a statistical test is robust, the validity of the test result will not be affected by poorly structured data. In other words, it is resistant against violations of parametric assumptions. Robustness has a more technical definition: If the actual Type I error rate of a test is close to the proclaimed Type I error rate, say 0.05 , the test is considered robust. In the context of Monte Carlo simulation, a robust model must be defined by a criterion of convergence or a specific cut-off value for the error rate [37, 38, 39]. The explanatory value of robustness study in statistics has been a controversial topic because both the initial conditions and the cut-off criterion are said to be artificial and arbitrary. Also, more seriously, robustness is a long run concept. This is not very impressive when certain values converge after running a large number of simulations. Some patterns would eventually emerge from a random, structured, or mixed process in the long run. It is impor- 
tant to point out that a mathematical model appealing to the long run is almost always right; it could explain many things but indeed its explanatory power is limited. For example, by invoking "regression towards the mean" [40] asserted that the returns in a financial time series can be very stable in the long run. If you are an investor, can you find anything useful from this statement? Borrowing [41] phrase "in the long run we are all dead", I would question the robustness criterion by saying "in the long run many things can converge." Further, when a robust model is imposed on specific data, there is a big logical gap between the general model and the particular data. It is legitimate to question how one could know that a robust model could be applied to the issue in question. In short, a stable model could be consistently wrong, and a robust model might have no connection to reality. As a matter of fact, the evolution of communication and establishing meaningful semantics is not fool-proof or certain. Failure of communication is a real possibility [42]. In a similar vein, [13] challenged Skyrms by arguing that we need to ask whether our ancestors really solved problems in the same manner as the games of divide-the-cake, the ultimate game, and the prisoners' dilemma. They contended that mathematical models are one thing, but empirical reality is another. Much more empirical work is needed to show how the Skyrmsian model and reality are connected. As a matter of fact, playing games in a controlled environment is vastly different from "playing games" in the real world. The dictator game, which is used very often for studying morality and cooperative behaviors, is a good example. In a typical study utilizing the dictator game, the first participant is told to divide a gift to the second participant. The game is so named because the decision made by the giver is final. At first glance, most results are encouraging: Many participants were willing to share the newly-acquired wealth. However, the result was completely different when the dictator game was conducted in a naturalistic setting. In a study [43] carried out at a bus stop in Las Vegas, the researcher told some strangers that he was in a hurry to the airport and therefore he wanted to give away his casino chips. The researcher explicitly suggested to the receivers to share a portion of the money to another stranger at the bus stop, who was actually a member of the research team. In contrast to the previous results, no one in the naturalistic study gave any portion of the endowment to the stranger. Put it bluntly, our realistic behaviors are context-dependent.

Further, [5] challenged the Kantian notion that lying is immoral but his example is unrealistic. According to Skyrms, in some games the average payoff with honest signaling is less than that with deceptive messaging. Thus, the player would systematically send deceptive signals, such as incomplete information or misinformation, to the other party. Although [44] praised this point as a logical counter-example against the Kantian notion, this argument is by no means new. First, many ethicists agree that in extraordinary situations it is imperative for us to lie. One classic example is that in 1943 the Allies implemented Operation Bodyguard to mislead the German intelligence about the strategic location of the D-Day invasion. Another good example also happened during World War II. When Jews were arrested and imprisoned in concentration camps, some courageous "Samaritans" lied to German soldiers looking for Jews hidden in their homes [45]. However, these are unusual times when not telling the truth is a good thing. It would be hard-pressed to imagine how a society could be better off without accepting honesty as the norm. Take China as an example. When too many manufacturers produce counterfeit, shoddy and dangerous products, at the end no one can tell which product is trustworthy. In this case it is selfdefeating for everyone to be dishonest for his or her own advantage [46]. [6] asserted that in some signaling games the sender always sends a signal that deceives the receiver. He wrote, "Universal deception in this strong sense is not only logically consistent in the sense of involving no contradiction, but also evolutionarily consistent in the sense of being an equilibrium...Kant was wrong, wasn't he?". However, the example used by Skryms is hypothetical: The player is locked in a cabinet and would like to be released. The only way for her to get out of the cabinet is to send signals to a receiver. According to [5], the best strategy should be sending deceptive signals all the time. Although this scenario is mathematically sound and logically possible, Skyrms failed to cite any realistic examples that can lead to any practical implications for improving social wellbeing.

Although it is doubtful whether the generalist account of evolutionary game theory could supersede the particularist account of evolutionary psychology, this does not imply that the Skyrmsian approach is fundamentally flawed or has no merit. On the contrary, mathematical modeling in evolutionary game theory is indispensable because it can account for the dynamic aspect of a model. To be explicit, it could effectively explain how a system could be destabilized and how an equilibrium could eventually be restored. As a matter of fact, the particularist account introduced by evolutionary psychology is not trouble-free. While evolutionary psychologists are proud of giving detail in their explanation, very often the detail can only explain a phenomenon in a specific setting; it fails to explain why changes in those norms and con- 
ventions occur later. For example, from the perspective of evolutionary psychology, men are pre-programmed, according to [47], to have as many women as possible in order to maximize the probability of procreation. It is noteworthy that Wright did not attempt to rationalize promiscuous sex or other egoistic behaviors; rather, he claimed that the aim of his analysis is to lead readers toward greater compassion and concern for their fellow human beings after exposing them to evolutionary psychology. However, if this aspect of so-called "human nature" had been hard-wired into every man's cognitive structure, it is very difficult to imagine how these static traits could be transformed, as they clearly would have to have been in many men. In addition, [48] argue that rape may have been favored by natural selection because it furthers male reproduction. In opposition to learning theory, which proposes that rape is a kind of unnatural and learned behavior through socialization, these evolutionary psychologists assert that rape is natural and genetically driven. It is important to point out that these authors do not justify rape by saying that it is natural. In contrast, they attempted to develop an evolutionarily informed educational program for young men that focuses on increasing their ability to restrain their sexual desire. However, regardless of how much psychological detail the theory illustrates, this theory is not applicable across all situations. Rape may be acceptable in a small number of societies, but in most cultures rape is considered both immoral and illegal. No convincing answer to why this is the case is offered by evolutionary psychologists.

As mentioned before, sociobiology is a precursor of evolutionary psychology, and not surprisingly, they face similar criticisms. During the 1970s, because of the perception that behaviors seem to be unalterable in his framework, E. O. Wilson, the founder of sociobiology, was accused of being a right-wing extremist who hijacked science to cover up racism and sex discrimination. Even if we put aside the agenda of political correctness, by the same token, evolutionary psychology also has difficulties in accounting for behavioral changes of a population. To be fair to evolutionary psychologists, they are not so naïve as to say that hard-wired psychological mechanisms exclude any cultural evolution and social learning. As mentioned previously, most evolutionary psychologists are not biological determinists. The issue is that the complexity of psychological mechanisms is a strong factor in evolutionary psychology; the more complex the mind is to start with, the more complex culture and society can become [49]. The more complex culture and society can become, the more resistance to change the society can face. In a modeling perspective, a high level of complex- ity creates tremendous difficulties in formulating a model of change. In this sense, evolutionary game theory seems to be a better analytical tool for the dynamic aspect of cultural evolution than evolutionary psychology, and thus it is inevitable for the former to sacrifice of certain detail.

\section{DISCUSSION}

Although evolutionary game theory has more potential for explaining mutation and change in population structure than its psychological counterpart has, no doubt there is a gap between the Skyrmsian simulation-based model and the reality. Nevertheless, the two approaches could work hand in hand to provide a comprehensive view of cultural evolution.

According to [50], there are two major approaches to viewing the nature of evolutionary theory, namely, the theory of force and the statistical interpretation. The following two scenarios were used to illustrate the differences between these two approaches. In the first scenario, a feather is dropped from a height of one meter. In the second, ten coins are randomly drawn from an urn containing 1000 coins: 500 with heads up, 500 tails up. In both cases we might make a prediction about the outcomes. We might predict that the feather will fall within a certain scope. In the coin-drawing case, we predict a distribution of heads and tails resulted from drawing. However, there is a significant difference between the two predictions. In the former, the trajectory of the falling feather is generated by the forces known by our physical laws. In the latter, the expected outcome does not result from attending to the forces acting on the coins. Instead, certain probability laws are at work, and the population structure must be taken into consideration. In other words, in the former case, particular details such as the force of gravity, the wind speed, the distance between the dropping point and the ground, and other conditions pertaining to physical laws should be taken into account to explain the outcome. In the latter the issue is purely mathematical and thus it is legitimate to apply mathematical criteria to evaluate the prediction. Furthermore, Walsh et al. asserted that the error types in these two approaches are totally different. In the case of falling feather, errors in prediction result from ignorance of detail, e.g., a scientist might not measure the wind speed correctly. However, in the coin-drawing scenario, the nature of the error is statistical, such as random fluctuations. There are two phenomena to be explained in evolution: The individual properties and the population structure, which are approached by some researchers from a theory of force and statistical explanation.

The above analogy could be well applied to the dis- 
tinction between evolutionary psychology and Skyrmsian mathematical modeling. The particularist account offered by evolutionary psychologists is a theory of force, in which specific causal agents, such as psychological drives, are said to bring out behavioral consequences. On the other hand, the Skyrmsian simulation-based approach is obviously a mathematically-based model, in which robustness can add weight to the explanatory power of a prediction. It is important to point out that the essence and purpose of these two types of theories are fundamentally different, and thus applying the criteria of one to dismiss another, or vice versa, is unfair and counterproductive.

It is not surprising that the evolutionary generalist approach is viewed as invoking "naturalistic God of the gaps" because many other statistical and probabilistic theories face the same kind of hostility. To be specific, in statistics most phenomena could be answered by blanket concepts like the following: "The score of group A is high in the first test and low in the second one because of random fluctuations." "The score is high in the first test and low in the second one because of regression to the mean." "Sometime the score is high and sometime it is low. This variation forms a normal curve. Many things in the world happen according to the bell curve effect." Needless to say, this kind of "universal" answer does not give any detail. Readers still have no clue why the test score is high in one test but low in another; what they need to know are the mental state of the test takers, the content of the items, and other relevant details. For a similar reason, philosopher of science [51] is critical of the explanatory power of the bell curve argument in the social sciences because the so-called bell curve effect has no causal power.

If using statistical laws is viewed as a convenient way to fill the explanatory gap, no wonder it is seen as a form of "naturalistic God of the gaps." Nevertheless, this criticism is unfair because the goal of statistics is to study the aggregate pattern of the subject matter with reference to some distributions, but not to illustrate the qualitative detail of the agents that cause changes in variables. By the same token, the Skyrmsian approach is not guilty of lacking detail and should reject the charge of invoking "naturalistic God of the gaps," for its merits are concerned with studying the structure of a population and the process of its change in a mathematical sense.

Hence, not only are the particular and the generalist approaches not mutually exclusive, but they also could be complementary to each other in the sense that the particularist account can specify input variables for a simulation-based methodology while the mathematical approach can evaluate the long run stability and the ro- bustness of a specified model. For example, the tendency of reciprocity among players is essential to the Skyrmsian game scenarios. In response to the Skyrmsian conception of morality, evolutionary psychologist Dennis Krebes elaborated on the behavioral pattern of reciprocity by incorporating insight from psychology. To be specific, [52] argued that evolutionary game theory could explain morality because we are biologically disposed to maximize others' tendency to practice the "Golden Rule" in their interactions with us. In other words, we preach this principle to create the impression that we practice it and expect the same to be done to us by others. Moreover, Krebes argued that humans inherit dispositions to invoke certain cooperative strategies that define moral development in Kohlberg's theory. In examining the robustness claim of the Skyrmsian model, [53] also suggested that for Skyrms's project to be carried through, the evolutionary models must be informed by the empirical work of evolutionary biologists, anthropologists, and primatologists. As mentioned in an earlier section, a stable and robust model may be a mis-specified model after all. A particular account can definitely be helpful in specifying a proper model. However, particularists such as psychologists, biologists, sociologists and anthropologists may provide too many diverse details to formulate a coherent global picture of cultural evolution. Also, a high level of complexity is detrimental to a model of change. The beauty of mathematics is in its ability to simplify the detail, suppress the noise, and smooth the residuals. When a positive feedback loop is formed between evolutionary psychology and evolutionary game theory, the chasm between modeling and reality might eventually be bridged.

\section{CONCLUSION}

The Skyrmsian approach to the emergence of signaling protocols and social networks is appealing because of its naturalness and spontaneity. This approach is said to a remedy for the shortcomings of other paradigms that necessitate rationality and recognition of salient features. Although some situations are rectified, new issues arise. Specifically, the Skyrmsian approach is phenotypic rather than genotypic because it focuses on mathematical topology without taking psychological dispositions into consideration. Unlike evolutionary psychology that investigates mental factors, the Skyrmsian approach is evolutionary generalist. His methodology is mathematical rather than empirical, but mathematical ideals might not be congruent with the real world. For example, Skyrms used a mathematically and logically sound scenario to illustrate how lying can be productive and why the Kantian notion of honesty is wrong, but it is doubtful whether 
his hypothetical example can result in any practical implications for improving social wellbeing. Additionally, a mathematical approach to the explanation of human behaviors is considered a kind of "naturalistic God of the gaps," because the so-called stable and robust results in the long run could explain too many things while the detail remains unexplained. Nevertheless, mathematical modeling in evolutionary game theory is still indispensable because it can account for the dynamic aspect of a model and compensate for the weakness of evolutionary psychology. Very often evolutionary psychologists fail to explain how and why certain norms and conventions could be broken. Nonetheless, it is the conviction of the author that the two approaches could work side by side to develop a comprehensive view of cultural evolution. In the future it is advisable for both evolutionary game theorists and evolutionary psychologists to extract robust behavioral patterns and social dynamics from big data $[54,55]$.

\section{REFERENCES}

[1] B. Skyrms, Evolution of the social contract. Cambridge, MA: Cambridge University Press, 1996.

[2] B. Skyrms, "Game theory, rationality and evolution of the social contract," Journal of Consciousness Studies, vol. 7, no. 1-2, pp. 269-284, 2000.

[3] B. Skyrms, "Stability and explanatory significance of some simple evolutionary models," Philosophy of Science, vol. 67, no. 1, pp. 94-113, 2000.

[4] B. Skyrms, The stag Hunt and the Evolution of Social Structure. Cambridge, MA: Cambridge University Press, 2004.

[5] B. Skyrms, "The flow of information in signaling games," Philosophical Studies, vol. 147, no. 1, pp. 155-165, 2010. doi: https://doi.org/10.1007/ s11098-009-9452-0

[6] B. Skyrms, Signals: Evolution, Learning, and Information. Oxford, UK: Oxford University Press, 2010.

[7] T. C. Schelling, The Strategy of Conflict. Cambridge, MA: Harvard University Press, 1963.

[8] A. S. Yee, "Thick rationality and the missing" brute fact": the limits of rationalist incorporations of norms and ideas," The Journal of Politics, vol. 59, no. 4, pp. 1001-1039, 1997.

[9] H. Gintis, "Classical versus evolutionary game theory," Journal of Consciousness Studies, vol. 7, no. 1-2, pp. 300-304, 2000.

[10] T. J. Brennan, "The rise of behavioral economics in regulatory policy: Rational choice or cognitive limitation?" International Journal of the Economics of Business, vol. 25, no. 1, pp. 97-108, 2018. doi: https://doi.org/10.1080/13571516.2017.1390833

[11] D. Kahneman and P. Egan, Thinking, Fast and Slow. New York, NY: Farrar, Straus and Giroux, 2011.

[12] D. Fred, Knowledge and the Flow of Information. Cambridge, MA: MIT Press, 1981.

[13] M. Barrett, E. Eells, B. Fitelson, and E. Sober, "Models and reality: A review of Brian Skyrms's evolution of the social contract," Philosophy and Phenomenological Research, vol. 59, no. 1, pp. 237-241, 1999.

[14] J. McEvoy, "Evolutionary game theory: Lessons and limitations, a cancer perspective," British Journal of Cancer, vol. 101, no. 12, pp. 2060-2061, 2009. doi: https://doi.org/10.1038/sj.bjc.6605444

[15] P. J. Vermeulen, J. Ruijven, N. P. Anten, and W. Werf, "An evolutionary game theoretical model shows the limitations of the additive partitioning method for interpreting biodiversity experiments," Journal of Ecology, vol. 105, no. 2, pp. 345-353, 2017. doi: https://doi.org/10.1111/ 1365-2745.12706

[16] J. D. Kelleher and B. Tierney, Data Science. Cambridge, MA: MIT Press, 2018.

[17] C. H. Yu, H. S. Lee, E. Lara, and S. Gan, "The ensemble and model comparison approaches for big data analytics in social sciences." Practical Assessment, Research \& Evaluation, vol. 23, no. 17, pp. 1-11, 2018.

[18] G. Mar, "Evolutionary game theory, morality and darwinism," Journal of Consciousness Studies, vol. 7, no. 1/2, pp. 322-325, 2000.

[19] S. Jones and E. Johnson, Psychology and Christianity: Five views. Downers Grove, IL: Intervarsity, 2010.

[20] N. Tennant, "Sex and the evolution of fair-dealing," Philosophy of Science, vol. 66, no. 3, pp. 391-414, 1999. doi: https://doi.org/10.1086/392694

[21] J. D’ Arms, R. Batterman, and K. Górny, "Game theoretic explanations and the evolution of justice," Philosophy of Science, vol. 65, no. 1, pp. 76-102, 1998. doi: https://doi.org/10.1086/392627

[22] W. B. Provine, The Origins of Theoretical Population Genetics: With a New Afterword. Chicago, IL: University of Chicago Press, 2001.

[23] C. H. Yu, Philosophical Foundations of Quantitative Research Methodology. Lanham, MD: University Press of America, 2006.

[24] P. W. Holland, "The future of evolutionary developmental biology," Nature, vol. 402, no. 6761supp, 
pp. C41-C44, 1999. doi: https://doi.org/10.1038/ 35011536

[25] V. Kvasnička and J. Pospí, "Emergence of modularity in genotype-phenotype mappings," Artificial Life, vol. 8, no. 4, pp. 295-310, 2002.

[26] D. M. Walsh, "Fit and diversity: Explaining adaptive evolution," Philosophy of Science, vol. 70, no. 2, pp. 280-301, 2003. doi: https://doi.org/10.1086/ 375468

[27] K. Schwenk, "Functional units and their evolution," in The Character Concept in Evolutionary Biology, G. P. Wagner, Ed. San Diego, CA: Academic Press, 2001, pp. 165-198.

[28] L. E. Harrison, Culture Matters: How Values Shape Human Progress. New York, NY: Basic books, 2002.

[29] M. Ruse and E. O. Wilson, "The evolution of ethics," New Scientist, vol. 108, no. 1478, pp. 50-52, 1985.

[30] C. Thongtawee, "Mask in performing arts: The change in spiritual and artistic value through evolution of social context," Journal of Advances in Humanities and Social Sciences, vol. 2, no. 2, pp. 98-106, 2016. doi: https://doi.org/10.20474/jahss-2. 2.4

[31] F. B. De Waal, "Evolutionary psychology: The wheat and the chaff," Current Directions in Psychological Science, vol. 11, no. 6, pp. 187-191, 2002. doi: https://doi.org/10.1111/1467-8721.00197

[32] J. D'Arms, "Sex, fairness, and the theory of games," The Journal of philosophy, vol. 93, no. 12, pp. 615-627, 1996. doi: https://doi.org/10.2307/ 2941119

[33] J. M. Alexander, "Evolutionary explanations of distributive justice," Philosophy of Science, vol. 67, no. 3, pp. 490-516, 2000. doi: https://doi.org/10. $1086 / 392792$

[34] J. Bradley. (2016) Gods dice: Randomness can have purpose. Christian century. [Online]. Available: https://bit.ly/2U7apdB

[35] C. H. Yu, "Beyond randomness and pattern: Are Christian and scientific worldviews compatible?" In Southern California Christians in Science Conference, Azusa, CA, 2014.

[36] C. H. Yu, "Test-Retest reliability," Encyclopedia of Social Measurement, vol. 3, pp. 777-784, 2005. doi: https://doi.org/10.1016/b0-12-369398-5/00094-3

[37] C. H. Yu, "An overview of remedial tools for violations of parametric test assumptions in the SAS system," In Proceedings of Western Users of SAS Software Conference, San Diego, CA, 2002.
[38] C. H. Yu. (2017) Exploratory data analysis. [Online]. Available: https://bit.ly/2CNYRoT

[39] W. I. Iryani and S. Y. Murtiwidayanti, "Empowering family with social-psychology problem through Family Care Unit (FCU)," International Journal of Humanities, Arts and Social Sciences, vol. 3, no. 2, pp. 53-63, 2017. doi: https://doi.org/10.20469/ijhss. 3.20003-2

[40] J. J. Siegel, Stocks for the long run: The definitive guide to financial market returns and long-term investment strategies. New York, NY: McGraw-Hill, 2007.

[41] J. M. Keynes, A Tract on Monetary Reform. London, UK: Macmillan, 2018.

[42] S. Huttegger, "Signals: Evolution, learning and information," Analysis, vol. 71, no. 3, pp. 597-599, 2011. doi: https://doi.org/10.1093/analys/anr054

[43] J. Winking and N. Mizer, "Natural-field dictator game shows no altruistic giving," Evolution and $\mathrm{Hu}$ man Behavior, vol. 34, no. 4, pp. 288-293, 2013. doi: https://doi.org/10.1016/j.evolhumbehav.2013. 04.002

[44] J. Z. Bonilla, "Signals: Evolution, learning, and information," THEORIA. An International Journal for Theory, History and Foundations of Science, vol. 27, no. 3, pp. 400-402, 2012. doi: https: //doi.org/10.1387/theoria.6582

[45] H. Varden, "Kant and lying to the murderer at the door... one more time: Kant's legal philosophy and lies to murderers and nazis," Journal of Social Philosophy, vol. 41, no. 4, pp. 403-421, 2010. doi: https://doi.org/10.1111/j.1467-9833.2010.01507.x

[46] P. Midler, Poorly Made in China: An Insider's Account of the China Production Game. Hoboken, NJ: John Wiley \& Sons, 2010.

[47] R. Wright, The Moral Animal: The New Science of Evolutionary Psychology. New York, NY: Pantheon Books, 1994.

[48] R. Thornhill and C. T. Palmer, A Natural History of Rape: Biological Bases of Sexual Coercion. Cambridge, MA: MIT Press, 2001.

[49] E. M. Gander, On Our Minds: How Evolutionary Psychology is Reshaping the Nature Versus Nurture Debate. Baltimore, MD: JHU Press, 2003.

[50] D. M. Walsh, T. Lewens, and A. Ariew, "The trials of life: Natural selection and random drift," Philosophy of Science, vol. 69, no. 3, pp. 429-446, 2002. doi: https://doi.org/10.1086/342454

[51] C. Glymour, "What went wrong? reflections on science by observation and The Bell Curve," Philos- 
ophy of Science, vol. 65, no. 1, pp. 1-32, 1998. doi: https://doi.org/10.1086/392624

[52] D. Krebs, "Evolutionary games and morality," Journal of Consciousness Studies, vol. 7, no. 1-2, pp. 313-321, 2000.

[53] Z. Ernst, "Explaining the social contract," The British journal for the philosophy of science, vol. 52, no. 1, pp. 1-24, 2001. doi: https://doi.org/10.1093/ bjps/52.1.1

[54] D. Bodoff, R. Bekkerman, and J. Dai, "Evolution of language: An empirical study at ebay big data lab," PloS one, vol. 12, no. 12, p. e0189107, 2017. doi: https://doi.org/10.1371/journal.pone.0189107

[55] Y. Zhao, D. Li, and L. Pan, "Cooperation or competition: An evolutionary game study between commercial banks and big data-based e-commerce financial institutions in China," Discrete Dynamics in Nature and Society, vol. 2015, 2015. doi: https://doi.org/10.1155/2015/890972 